\title{
e-Phaïstos
}

e-Phaïstos Revue d'histoire des techniques / Journal of the history of technology

IX-1 | 2021

Autour de Léonard de Vinci

\section{Le Léonard de Paul Valéry}

Paul Valéry's Leonardo da Vinci

\section{Antoine Valéry}

\section{OpenEdition}

\section{Journals}

Édition électronique

URL : https://journals.openedition.org/ephaistos/8858

DOI : 10.4000/ephaistos.8858

ISSN : 2552-0741

Éditeur

IHMC - Institut d'histoire moderne et contemporaine (UMR 8066)

\section{Référence électronique}

Antoine Valéry, «Le Léonard de Paul Valéry », e-Phaïstos [En ligne], IX-1 | 2021, mis en ligne le 27 avril 2021, consulté le 17 septembre 2021. URL : http://journals.openedition.org/ephaistos/8858 ; DOI : https://doi.org/10.4000/ephaistos.8858

Ce document a été généré automatiquement le 17 septembre 2021.

Tous droits réservés 


\title{
Le Léonard de Paul Valéry
}

\author{
Paul Valéry's Leonardo da Vinci
}

Antoine Valéry

1 Ingénieur, inventeur, anatomiste, peintre, sculpteur, architecte, urbaniste, botaniste, musicien, poète, philosophe et écrivain, Léonard de Vinci s'est, on le sait, illustré dans tous les domaines de l'art et des sciences. Mais au-delà du caractère encyclopédique de son œuvre, il y a chez Léonard de Vinci une véritable recherche quant à l'élaboration de la pensée qui, quelques siècles plus tard, va fasciner Paul Valéry pour lequel il s'agira d'une préoccupation constante. C'est ainsi que le futur auteur de La jeune Parque et du Cimetière marin publie en 1895 un essai intitulé Introduction à la méthode de Léonard de Vinci qu'il reprendra à plusieurs reprises et ceci tout au long de sa vie.

\section{Le contexte intellectuel de 1895}

\section{Dans l'histoire des sciences, des arts et de la pensée}

2 Quelques événements peuvent servir de points de repères. Sur le plan scientifique, le physicien allemand Wilhelm Röntgen découvre les rayons $\mathrm{X}$ pendant que Marconi invente le télégraphe sans fil. Avec les frères Lumière vient le temps du cinématographe. Édouard Michelin invente le pneumatique démontable et Albert Calmette met au point les premiers anti-venins. En architecture, l'édification du Reichstag de Berlin se poursuit alors que celle du Tower Bridge de Londres débute l'année suivante. En sculpture, le premier exemplaire des Bourgeois de Calais, d'Auguste Rodin, est inauguré. Parmi les publications essentielles, on peut citer les Règles de la méthode sociologique d'Émile Durkheim et les premiers travaux de Freud.

3 La période, les années 1890, correspond à un regain d'intérêt pour Léonard de Vinci. Il est notamment dû à la publication par Charles Ravaisson-Mollien du Codex sur le vol des oiseaux, du Traité de la peinture et des Manuscrits en six volumes (fac-similé, transcription littérale et traduction française). Valéry sera fasciné par le caractère encyclopédique de ces manuscrits. À ce propos, il note : «Si légèrement que je l'eusse étudié, ses dessins, ses manuscrits m'avaient comme ébloui. De ces milliers de notes et 
de croquis, je gardais l'impression extraordinaire d'un ensemble hallucinant d'étincelles arrachées par les coups les plus divers à quelque fantastique fabrication " (Valéry $2016: 825$ ).

4 Où en est Paul Valéry en 1895 ? Il a 23 ans. Ayant commencé en 1890 ses études de droit à Montpellier, il s'est initié aux mathématiques grâce à son ami Pierre Féline qui prépare Polytechnique et a déjà écrit et publié plusieurs poèmes. Trois ans après « La Nuit de Gênes " pendant laquelle il se remet en cause - «je me sens autre $»^{1}$ - il s'est installé à Paris et travaille au ministère de la guerre. Valéry commence à tenir ses Cahiers qu'il poursuivra jusqu'à la fin de sa vie et dont les nombreuses notations scientifiques et mathématiques témoignent déjà de l'éclectisme de ses recherches.

5 En effet, il a déjà lu le Dictionnaire raisonné de l'architecture française de Viollet-le-Duc "L'architecture a tenu une grande place dans les premières amours de mon esprit », la Grammaire de l'ornement d'Owen Jones, l'Histoire de l'art monumental de Louis Batissier, le Traité d'électricité et de magnétisme de Maxwell, le traité Constitution de la matière de William Thomson, les articles du mathématicien Henri Poincaré qui a publié en 1893 Les méthodes nouvelles de mécanique céleste. D'ailleurs, il fréquentera plus tard de très grands scientifiques tels que les physiciens Louis et Maurice de Broglie, Marie Curie, Louis Leprince-Ringuet et Jean Perrin ainsi que le mathématicien Émile Picard et même Albert Einstein.

\section{Pourquoi Léonard de Vinci pour Valéry?}

6 L'Introduction à la méthode de Léonard de Vinci a pour origine une commande d'article de Juliette Adam, femme de lettres, fondatrice de La Nouvelle Revue, qui encourage les débuts littéraires de Pierre Loti, d'Alexandre Dumas fils et de Léon Daudet (Valéry 2016 :823). Valéry est conscient de la difficulté : «Je me propose d'imaginer un homme de qui auraient paru des actions tellement distinctes que si je viens à leur supposer une pensée, il n'y en aura pas de plus étendue » (Valéry $2016: 120)$. «Je me fiche du côté critique d'art et d'érudition. J'écarte le Vinci connu et je veux établir un modèle (dans le sens de modèle mécanique) de l'esprit d'un Vinci, les conditions du problème étant pour moi réduites à ce point ${ }^{2}$ "; "J'ai à combiner les termes suivants, peinture, architecture, mathématiques, mécanique, physique et mécanismes » (Valéry 1974 :22).

\section{Que représente Léonard pour Valéry?}

7 C'est un homme d'esprit universel. Pour Valéry, c'est « d'un mot, l'artiste du monde » (Valéry $2016: 825)$. «Léonard - pressentiment de l'ère moderne », écrira-t-il dans ses Cahiers $^{3}$. En fait, Vinci illustre et conforte la vision des sciences de Valéry, par opposition à la philosophie: "La philosophie est comme mortifiée par l'immense distance qui s'est peu à peu créée entre ses résultats vagues et invérifiables et ceux des sciences (Valéry $2016: 857$ ).

Illustrons ce propos à partir de quelques exemples choisis parmi tant d'autres dans différentes disciplines :

Mathématiques : « La mathématique est la description des opérations mentales en tant qu'on peut les noter exactement... 》 (Valéry $2016: 783)$; «La science des machines de l'esprit, des machines qui sont dans l'esprit » (Valéry $206: 791$ ). 
10 Géométrie : «L'important et le beau de la géométrie, c'est (par sa pureté) qu'elle est un instrument de pensée - un mode de traitement - une manière de voir et de prolonger ou non un objet étranger » (Valéry 2016 :783).

11 Architecture : «Comme mon esprit s’y retrouve », écrira-t-il (Valéry 2016 :943); ce que confirment ses dialogues Eupalinos ou l'architecte de 1921 (Valéry 2016 :473).

12 Science : Pour Valéry, la science est un «tas de suppositions dont on recherche les résistances ${ }^{4} »$; «Une science réelle, écrit-il, n'est pas un système de réponses. Au contraire, c'est un système de problèmes qui demeurent toujours ouverts » (Valéry 2016) ; "Certains travaux des sciences, par exemple, et ceux des mathématiques en particulier, présentent une telle limpidité de leur armature qu'on les dirait l'œuvre de personne » (Valéry $2016: 870)$.

13 Il croit en la force de la science par rapport à ce que l'on croit acquis : «Le génie de Galilée a consisté un jour à oser penser contre l'expérience » (Valéry 1974 :843).

En fait, Vinci lui apparaît comme l'homme complet. C'est la pensée même, le créateur de ce que l'on appelle aujourd'hui l'interdisciplinarité. Comme lui, Valéry questionne quantité de disciplines : «La spécialité m'est impossible, reconnaît-il. Je fais sourire. Vous n'approfondissez rien. De quel droit parlez-vous de ceci à quoi vous n'êtes pas exclusivement consacré ? Ma spécialité, c'est mon esprit » (Valéry 2016 :77).

15 C'est aussi l'homme univers : "L'homme emporte ainsi des visions, dont la puissance fait la sienne" (Valéry $2016: 124)$; «L'homme universel commence, lui aussi, par contempler simplement, et il revient toujours à s'imprégner de spectacles. Il retourne aux ivresses de l'instant particulier et à l'émotion que donne la moindre chose réelle, quand on les regarde tous deux, si bien clos par toutes leurs qualités et concentrant de toute manière tant d'effets » (Valéry 2016 :879).

C'est « l'Homme d'Univers » qu'il ne faut pas confondre avec le « Grand homme » :

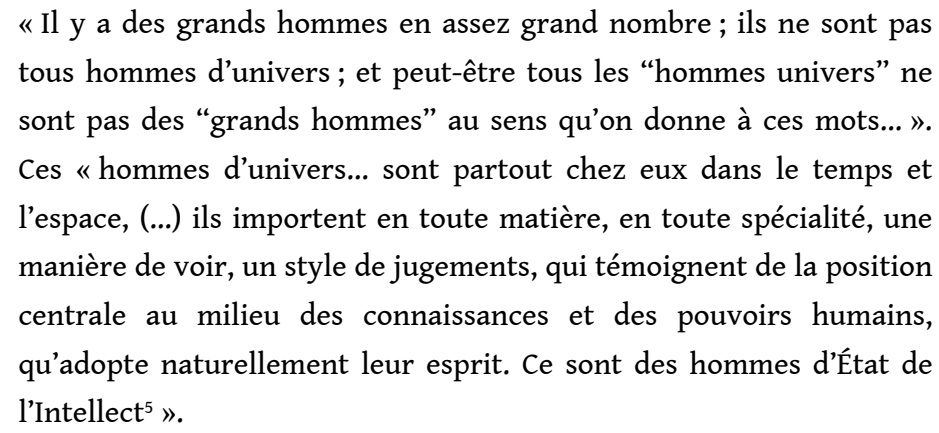

l'Intellect ${ }^{5}$ ».

17 Mais Léonard ne peut pas se réduire à son œuvre, si immense soit-elle.

18 Léonard, pour Valéry, c'est aussi et surtout le « faire ». Valéry souligne les limites de l'art : « Une œuvre très belle est celle qui pendant un temps donne l'impression d'être l'unique objet, - l'indispensable, le véritable. Et plus ce temps est grand, plus elle est belle. Mais je sais qu'il est toujours fini» (Valéry 1974 :925); «La conscience des opérations de la pensée, qui est la logique méconnue dont j'ai parlé, n'existe que rarement, même chez les plus fortes têtes " (Valéry 2016 :875). Le processus de la création est plus important à ses yeux que la création elle-même : "L'action qui fait plus que le chose faite» (Valéry 2016 :1343). Pour le poète qu'il est, «Être poète, non. Pouvoir l'être » (Valéry 2016 :20). 
19 La leçon inaugurale de son cours de "poïétique » au Collège de France en 1937 est éclairante : «Un nom manque à cette créature de pensée, pour contenir l'expansion de termes trop éloignés d'ordinaire et qui se déroberaient. Aucun ne me paraît plus convenir que celui de Léonard de Vinci » (Valéry 2016:212). Il «a fixé devant son regard cette Rigueur Obstinée, qui se dit elle-même la plus exigeante de toutes" (Valéry $2016: 833$ ). "Léonard, de recherche en recherche, se fait très simplement toujours plus admirable écuyer de sa propre nature ; il dresse indéfiniment ses pensées, exerce ses regards, développe ses actes » (Valéry $2016: 837$ ).

\section{Derrière Léonard, c'est Valéry lui-même}

20 "Je veux être maître chez moi ", écrit-il en 1894 à son ami André Gide (Valéry 2016: 19). Il s'interroge sur la valeur de l'œuvre d'art : «Léonard ou la spéculation vérifiée par la construction. La vraie valeur de l'œuvre d'art est peut-être de donner valeur à la spéculation, de trouver les fabrications qui soient à la spéculation ce que les résultats pratiques sont à la géométrie, à la mécanique » (Valéry 1974 :2019).

21 Pour Valéry, Léonard est une sorte de reflet de son propre esprit: "La nécessité où j'étais placé, le vide que j'avais si bien fait de toutes les solutions antipathiques à ma nature, l'érudition écartée, les ressources rhétoriques différées, tout me mettait dans un état désespéré... Enfin, je le confesse, je ne trouvai pas mieux que d'attribuer à l'infortuné Léonard mes propres agitations, transportant le désordre de mon esprit dans la complexité du sien. Je lui infligeai tous mes désirs à titre de choses possédées. Je lui prêtai bien des difficultés qui me hantaient dans ce temps-là, comme s'il les eût rencontrées et surmontées. Je changeai mes embarras en sa puissance supposée. J'osai me considérer sous son nom, et utiliser ma personne » (Valéry $2016: 861$ ).

«Que peut un homme?» (Valéry 2016:1025), se demande Valéry dans La soirée avec Monsieur Teste. La devise de Léonard de Vinci, "Hostinato rigore: obstinée rigueur " (Valéry 2016 : 868) n'est-elle pas une réponse, la réponse à cette interrogation?

\section{BIBLIOGRAPHIE}

VALÉRY Paul, « Introduction à la méthode de Léonard de Vinci », Euvres, tome 1, Paris, La Pochothèque, 2016

VALÉRY Paul, Cahiers, tome 2, Paris, La Pléiade, 1974

\section{NOTES}

1. Manuscrit, Bibliothèque littéraire Jacques Doucet, Valeryanum, cité par Françoise Haffner, Sous une si grande ombre ... Valéry et le fantôme de Mallarmé, Presses universitaires de Perpignan, 2011. 
2. Lettre à sa mère citée par Michel Jarrety in Paul Valéry, Euvres, La Pochothèque t.1, 2016, p. 109.

3. Cahiers, La Pléiade, t.2, p.861

4. Cahiers, La Pléiade, t.2, p.833

5. Entretiens sur Goethe, Francfort, 12-14 mai 1932

\section{RÉSUMÉS}

La fin du XIX siècle correspond à un regain d'intérêt pour l'œuvre multiforme de Léonard de Vinci. À côté des historiens de l'art, des philosophes et des savants qui se sont passionnés, voire déchirés pour le génie italien, Paul Valéry occupe une place à part. Très tôt, il s'est plongé dans les publications des manuscrits de Léonard. Il devait en tirer une œuvre de jeunesse, Introduction à la méthode de Léonard de Vinci. Ce texte saisissant, évolutif, a accompagné Paul Valéry tout au long de son parcours d'écrivain, tout en rencontrant un accueil particulier et même en exerçant une véritable fascination chez certains ingénieurs $\mathrm{du} \mathrm{XX}$ siècle. C'est la première version de $L a$ Méthode, celle parue en 1895, que cet article propose de présenter.

The end of the 19th century saw a renewed interest in the multifaceted work of Leonardo da Vinci. Alongside intellectuals who were passionate about the Italian genius, Paul Valéry occupies a special place. Very early on, he dived into the Leonardo's manuscripts, from which he wrote his first book, Introduction à la méthode de Léonard de Vinci. Paul Valéry worked on this text throughout his entire life. The Introduction fascinated the engineers who often drew inspiration from it in their design works. It is the first version of the book, published in 1895, which is presented here.

\section{INDEX}

Mots-clés : histoire des techniques, histoire des sciences, histoire de la littérature, interdisciplinarité

Keywords : history of technology, history of science, history of literature, Leonardo da Vinci, interdisciplinarity

\section{AUTEUR}

\section{ANTOINE VALÉRY}

Avocat honoraire au Barreau de Paris 